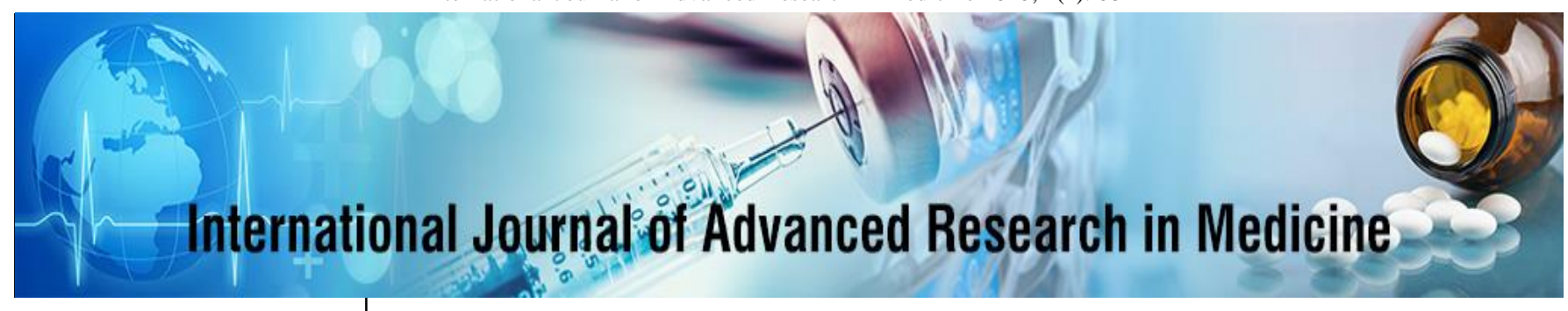

E-ISSN: 2706-9575 P-ISSN: 2706-9567 IJARM 2020; 2(1): 08-11 Received: 05-01-2020 Accepted: 06-02-2020

Dr. Mohd Akram Qureshi MD Pulmonary Medicine, Bidar Institutes of Medical Science, Bidar, Karnataka, India

\section{Correlation between presence of nocturnal oxygen desaturation with pulmonary hypertension}

\author{
Dr. Mohd Akram Qureshi \\ DOI: $\underline{\text { https://doi.org/10.22271/27069567.2020.v2.i1a.30 }}$
}

\begin{abstract}
Background: Chronic obstructive pulmonary disease (COPD) is the fourth leading cause of mortality and its prevalence continues to increase. While the debilitating effects of COPD on daytime functioning are well known, COPD's effects on sleep have been less fully investigated. Sleep problems affect as many as $50 \%$ of patients with COPD. In patients with mild and moderate COPD, studies on the relationship between pulmonary hypertension and NOD are not conclusive and their interpretation depends on the type of definition used for NOD, as well as the amount of decrease in $\mathrm{PaO} 2$.

Aim of the study: To study presence of nocturnal oxygen desaturation with pulmonary hypertension. Materials and methods: For the study, a total of 100 patients attending the outpatient department of pulmonary medicine diagnosed with moderate to severe COPD were selected. The patient's data was collected over a period of one year. Patients having comorbidities such as cardiovascular diseases, sleep apnea, bronchial asthma, morbid obesity were excluded from the study. An informed written consent was obtained from all the participants after explaining them the protocol of the study verbally. Data was collected from the participants such as clinical history of COPD, smoking history. For nocturnal oximetry, measurement of oxygen saturation was done at night from 11:00 pm to 07:00 am. Results: The mean age of patients was 69.51 years. The mean duration of COPD was 4.14 years. The mean smoking index of study population was 401.32. Mean baseline SPO2. The mean BMI was 26.32 $\mathrm{kg} / \mathrm{m} 2$. The mean hemoglobin level was $11.21 \mathrm{~g} / \mathrm{dL}$. The partial pressure of oxygen and carbon dioxide in desaturators and nondesaturators was $61.54 \mathrm{mmHg}$ and $70.65 \mathrm{mmHg}$ respectively. The presence of nocturnal hypoxemia has been postulated as the etiopathological factor resulting in the development of pulmonary hypertension in COPD patients.

Conclusion: From the results of the present study, this can be concluded that $29 \%$ of the normoxemic patients had significant nocturnal desaturation. The significant predictors of nocturnal desaturation are $\mathrm{PaO} 2$ and $\mathrm{PaCO} 2$.
\end{abstract}

Keywords: COPD, pulmonary hypertension, oxygen saturation

\section{Introduction}

Chronic obstructive pulmonary disease (COPD) is the fourth leading cause of mortality and its prevalence continues to increase ${ }^{[1]}$. While the debilitating effects of COPD on daytime functioning are well known, COPD's effects on sleep have been less fully investigated. Sleep problems affect as many as $50 \%$ of patients with COPD ${ }^{[2]}$. The mild hypoventilation that is physiologically observed during sleep is heightened in this patient group and is often accompanied by ventilation/perfusion mismatch ${ }^{[3]}$. In patients with mild and moderate COPD, studies on the relationship between pulmonary hypertension and NOD are not conclusive and their interpretation depends on the type of definition used for NOD, as well as the amount of decrease in $\mathrm{PaO} 2^{[4-6]}$. Hence, the present study was conducted to study presence of nocturnal oxygen desaturation with pulmonary hypertension.

\section{Materials and methods}

For the study, a total of 100 patients attending the outpatient department of Pulmonary medicine diagnosed with moderate to severe COPD were selected. The patient's data was collected over a period of one year. Patients having comorbidities such as cardiovascular diseases, sleep apnea, bronchial asthma, morbid obesity were excluded from the study. An informed written consent was obtained from all the participants after explaining them the protocol of the study verbally. Data was collected from the participants such as clinical history of COPD, smoking history. For nocturnal oximetry, measurement of oxygen saturation was done at night from 11:00 pm to 07:00 am in the hospital using MIR Spiro bank II with a transcutaneous finger probe while patient is breathing environmental air.
Corresponding Author: Dr. Mohd Akram Qureshi MD Pulmonary Medicine, Bidar Institutes of Medical Science, Bidar, Karnataka, India 
The statistical analysis of the data was done using SPSS version 11.0 for windows. Chi-square and Student's t-test were used for checking the significance of the data. A pvalue of 0.05 and lesser was defined to be statistical significant.

\section{Results}

Table 1 shows demographic data of the patients at baseline. The mean age of patients was 69.51 years. The mean duration of COPD was 4.14 years. The mean smoking index of study population was 401.32. Mean baseline SPO2. The mean BMI was $26.32 \mathrm{~kg} / \mathrm{m} 2$. The mean hemoglobin level was $11.21 \mathrm{~g} / \mathrm{dL}$. Table 2 shows factors associated with nocturnal oxygen desaturation. We observed that 29 patients had significant desaturation. The partial pressure of oxygen and carbon dioxide in desaturators and nondesaturators was
$61.54 \mathrm{mmHg}$ and $70.65 \mathrm{mmHg}$ respectively. The presence of nocturnal hypoxemia has been postulated as the etiopathological factor resulting in the development of pulmonary hypertension in COPD patients. On comparing the results were seen to be statistically significant with respect to partial pressure of oxygen and carbon dioxide.

Table 1: Demographic data of the patients at baseline

\begin{tabular}{|c|c|c|}
\hline Variables & Mean values & p-value \\
\hline Age (years) & 69.51 & 0.2 \\
\hline Duration of COPD (years) & 4.14 & 0.26 \\
\hline Smoking index $(\mathrm{n}=81)$ & 401.32 & 1.65 \\
\hline Baseline SPO2\% & 93.26 & 1.7 \\
\hline BMI $\left(\mathrm{kg} / \mathrm{m}^{2}\right)$ & 26.32 & 0.5 \\
\hline Hemoglobin g/dL & 11.21 & 0.2 \\
\hline
\end{tabular}

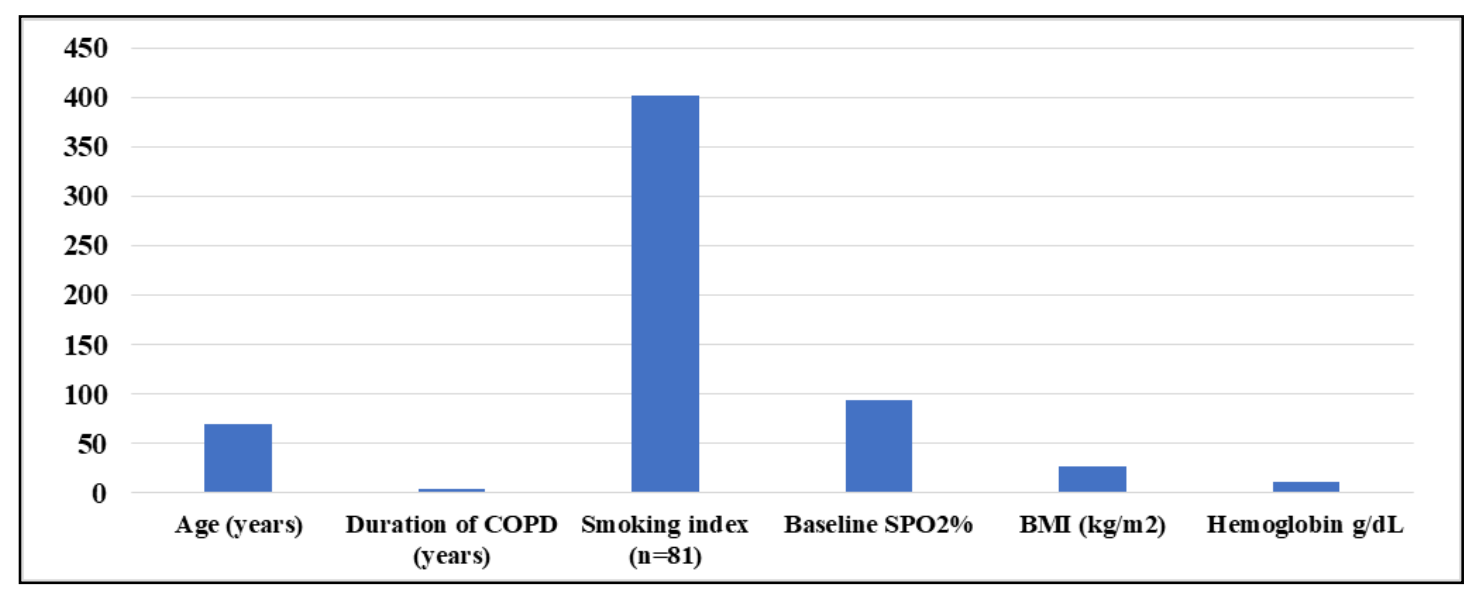

Fig 1: Demographic data

Table 2: Factors associated with nocturnal oxygen desaturation

\begin{tabular}{|c|c|c|c|}
\hline & \multicolumn{2}{|c|}{ Desaturation } & \multirow{2}{*}{ p-value } \\
\cline { 2 - 4 } & Desaturators (n=29) & Non-De saturators (n=71) & 0.21 \\
\hline Duration of COPD (years) & 3.96 & 3.41 & 0.06 \\
\hline Baseline SPO2\% & 94.36 & 94.32 & 0.12 \\
\hline $\mathrm{BMI}\left(\mathrm{kg} / \mathrm{m}^{2}\right)$ & 23.65 & 25.58 & 0.33 \\
\hline $\mathrm{Hemoglobin}(\mathrm{g} / \mathrm{dL})$ & 12.62 & 13.25 & $<0.001$ \\
\hline $\mathrm{PaO}_{2}(\mathrm{mmHg})$ & 61.54 & 70.65 & $<0.001$ \\
\hline $\mathrm{PaCO}_{2}(\mathrm{mmHg})$ & 53.25 & 40.16 & \\
\hline
\end{tabular}

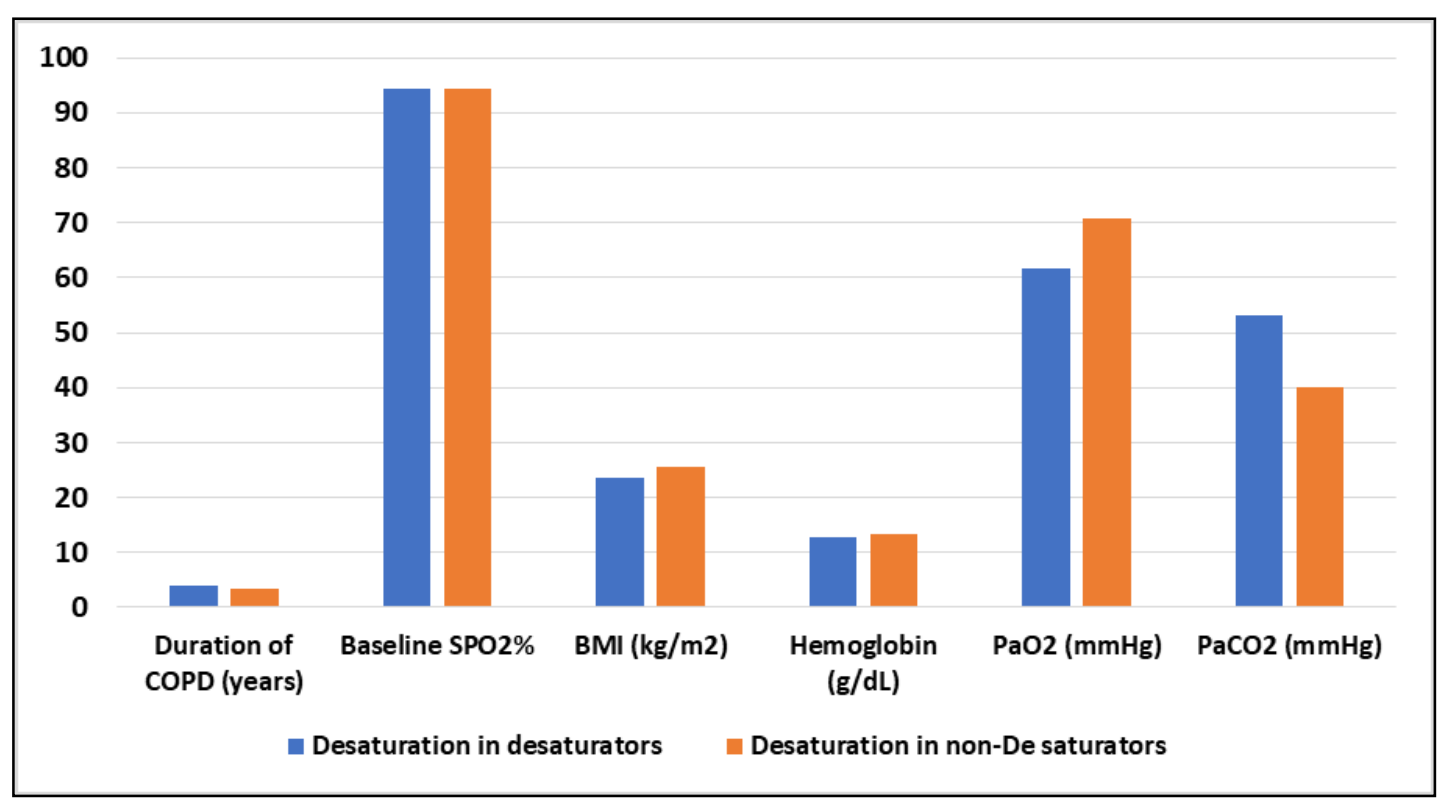

Fig 2: Nocturnal oxygen desaturation 


\section{Discussion}

In the present study, we observed that the partial pressure of oxygen and carbon dioxide in desaturators and nondesaturators was $61.54 \mathrm{mmHg}$ and $70.65 \mathrm{mmHg}$ respectively. The presence of nocturnal hypoxemia has been postulated as the etiopathological factor resulting in the developments of pulmonary hypertension in COPD patients. On comparing the results were seen to be statistically significant with respect to partial pressure of oxygen and carbon dioxide. The results were compared with previous studies from the literature and were found to be consistent with the results. Minai OA et al., determined the prevalence and significance of nocturnal oxygen desaturation in patients with PAH. A cross-sectional study conducted at the Cleveland Clinic. Patients were followed up at our institution except for the overnight oximetry study done at home. Data regarding degree of nocturnal desaturation, demographics, hemodynamics, pulmonary function, and functional capacity were collected. Forty-three patients (mean age, $47.9+/-13.5$ years [+/- SD]; 36 women and 7 men) underwent nocturnal oximetry. The etiology of PAH included idiopathic PAH (88\%) and PAH associated with connective tissue diseases $(12 \%)$. The majority of patients were New York Heart Association functional class II (42\%) or III $(53 \%)$. Thirty patients $(69.7 \%)$ spent $>10 \%$ of sleep time with oxygen saturation by pulse oximetry $<90 \%$. Desaturators were older $(\mathrm{p}=0.024)$ and had higher hemoglobin $(\mathrm{p}=0.002)$. Sixteen of 27 patients $(59 \%)$ without desaturation $<90 \%$ during a 6 -min walk test were nocturnal desaturators. Nocturnal desaturators had higher brain natriuretic protein, lower cardiac index, and higher mean right atrial pressure, mean pulmonary artery pressure, and pulmonary vascular resistance. On echocardiography, desaturators were more likely to have moderate or severe right ventricular dilation and pericardial effusion. Only one patient had significant sleep apnea. They concluded that nocturnal hypoxemia is common in PAH patients and correlates with advanced pulmonary hypertension and right ventricular dysfunction. Approximately 60\% patients without exertional hypoxia had nocturnal desaturation. Overnight oximetry should be considered in the routine workup of PAH patients who do not demonstrate exertional desaturation. Halank $\mathrm{M}$ et al., retrospectively analyzed patients with PPHT, in whom right heart catheterization, pulmonary function testing, measurements of arterial oxygen tension $(\mathrm{paO}(2))$ during daytime both at rest and at maximum physical exercise on cycle ergometer, and nocturnal polygraphy including pulse oximetry were performed. The data of these examinations were analyzed. Six patients with moderate to severe PPHT were included. Mean pulmonary artery pressure was $50+/-8 \mathrm{mmHg}$. Lung function did not reveal relevant pathological findings. Pulse oximetry demonstrated moderate to severe NOS as classified by duration of oxygen desaturation $<90 \%$ in 5 patients. $\mathrm{Mean} \mathrm{paO}(2)$ at rest and during maximum exercise was $10.6+/-1.2 \mathrm{kPa}$ and $9.2+/-1.6 \mathrm{kPa}$, respectively. One of 5 patients with moderate to severe NOS demonstrated exertional hypoxemia with a $\mathrm{paO}(2)$ value of $<8.0 \mathrm{kPa}$. None of the patients showed sleep apnea. They concluded that moderate to severe nocturnal hypoxemia seems to be common and is not related to sleep apnoea and lung function in patients with PPHT. The absence of exertional hypoxemia does not exclude NOS. We recommend to perform overnight oximetry in the routine examination of
PPHT patients because nocturnal oxygen supplementation should be considered in patients with NOS ${ }^{[7,8]}$.

Lacasse $\mathrm{Y}$ et al., characterized the perception and clinical practice of Canadian respirologists regarding the indications and prescription of nocturnal oxygen therapy in COPD, and to determine what Canadian respirologists consider an important treatment effect of nocturnal oxygen therapy in a randomized, placebo-controlled trial. A mail survey of all the respirologists registered in the 2006 Canadian Medical Directory was conducted. A total of 543 physicians were surveyed. The response rate was $60 \%$, and $99 \%$ of the respondents indicated that the problem of nocturnal oxygen desaturation is clinically relevant. Eighty-two per cent interpret oximetry tracings themselves, and $87 \%$ have access to a sleep laboratory. Forty-two per cent believe that all COPD patients with significant nocturnal desaturation should have a polysomnography to rule out sleep apnea, and $41 \%$ would prescribe nocturnal oxygen therapy to active smokers. Assuming a risk of death or progression to LTOT of $40 \%$ over a three-year period, the respirologists indicated that to declare nocturnal oxygen therapy effective in reducing the rate of major clinical events in a clinical trial, the minimal absolute risk difference of death or progression to LTOT between oxygen and room air breathing should be 14\%. They conlcluded that Canadian respirologists are interested in the issue of nocturnal oxygen desaturation in COPD. There is variation in clinical practices among Canadian respirologists in several aspects of the management of this problem. Toraldo DM et al., refined the concept of phenotyping desaturators in COPD and shows a possible pattern which could be used as a framework for future research. COPD is a complex condition with pulmonary and extrapulmonary manifestations. They suggested that COPD phenotypes should be associated with clinically meaningful outcomes. The innovation of COPD phenotyping is defined as COPD desaturators. Sleep-related hypoxemia and hypercapnia are well recognized in COPD and the development of systemic inflammation during sleep. These sleep-related changes predispose to nocturnal cardiac arrhythmias, pulmonary hypertension, and possibly death, particularly during acute exacerbations. They concluded that a more focused definition makes possible a classification of patients into two distinct subgroups for both clinical and research purposes. Establishing a common language for future research will facilitate understanding and management of such diseases. Even if different treatment strategies have different outcomes for these groups, we will have confirmation, or otherwise, of the clinical value of cluster analysis. This knowledge could lead to pharmacological treatment and other interventions directed to specific phenotypic groups ${ }^{[9,10]}$.

\section{Conclusion}

From the results of the present study, this can be concluded that $29 \%$ of the normoxemic patients had significant nocturnal desaturation. The significant predictors of nocturnal desaturation are $\mathrm{PaO} 2$ and $\mathrm{PaCO} 2$.

\section{References}

1. Fletcher EC, Luckett RA, Miller T, et al. Pulmonary vascular hemodynamics in chronic lung disease patients with and without oxyhemoglobin desaturation during sleep. Chest. 1989; 95:757-64.

2. Levi-Valensi P, Weitzenblum E, Rida Z, et al. Sleep- 
related oxygen desaturation and daytime pulmonary haemodynamics in COPD patients. Eur Respir J. 1992; 5:301-7.

3. Wright JL, Levy RD, Churg A. Pulmonary hypertension in chronic obstructive pulmonary disease: current theories of pathogenesis and their implications for treatment. Thorax. 2005; 60:605-9.

4. Abraham AS, Cole RB, Green ID. Factors contributing to the reversible pulmonary hypertension in patients with acute respiratory failure studied by serial observations during recovery. Circ Res. 1969; 24:5160.

5. Alp S, Skrygan M, Scmidt WE, et al. Sildenafil improves hemodynamic parameters in COPD- an investigation of six patients. Pulm Pharmacol Ther. 2006; 19:386-90.

6. Andreas S, Hermann-Lingen $\mathrm{C}$, Raupach $\mathrm{T}$, et al. Angiotensin II blockers in obstructive pulmonary disease. A randomized, controlled trial. Eur Respir J. 2006; 27:972-9.

7. Minai OA, Pandya CM, Golish JA, et al. Predictors of nocturnal oxygen desaturation in pulmonary arterial hypertension. Chest. 2007; 131(1):109-117. doi:10.1378/chest.06-1378

8. Halank M, Langner S, Kolditz M, Miehlke S, Höffken G. Nocturnal oxygen desaturation is a frequent complication in portopulmonary hypertension. Z Gastroenterol. 2008; 46(11):1260-1265. doi:10.1055/s2008-1027526

9. Lacasse Y, Sériès F, Martin S, Maltais F. Nocturnal oxygen therapy in patients with chronic obstructive pulmonary disease: a survey of Canadian respirologists. Can Respir J. 2007; 14(6):343-348. doi:10.1155/2007/487831

10. Toraldo DM, De Nuccio F, Gaballo A, Nicolardi G. Use of cluster analysis to describe desaturator phenotypes in COPD: correlations between pulmonary function tests and nocturnal oxygen desaturation. Int $\mathbf{J}$ Chron Obstruct Pulmon Dis. 2011; 6:551-561. doi:10.2147/COPD.S25383 